\title{
Complementary and alternative medicine in patients with inflammatory bowel disease: hype or evidence?
}

\author{
Jae Myung Cha \\ Department of Internal Medicine, Kyung Hee University School of Medicine, Seoul, Korea
}

\section{Article: Changes in prevalence and perception of complementary and alternative medicine use in Korean inflammatory bowel disease patients: results of an 8-year follow-up survey (Intest Res 2020;18:192-199)}

Complementary and alternative medicine (CAM) can be defined as interventions neither taught widely in medical schools nor generally available in medical hospitals. ${ }^{1}$ It has attracted increased public attention from the media, the Oriental medical community, and governmental agencies. The use of CAM has been increasing worldwide over the past two decades, ${ }^{2,3}$ and an estimated $21 \%$ to $77 \%$ of IBD patients use $\mathrm{CAM}^{4-6}{ }^{4-6} \mathrm{How}-$ ever, majority of previous studies were cross-sectional, and only one Manitoba cohort study reported the longitudinal trend of CAM use in IBD patients. ${ }^{7}$ In the present issue of Intestinal Research, Lee et al. ${ }^{8}$ reported the changes in the prevalence of CAM use, patterns of CAM use, and the perception and attitude towards CAM in Korean IBD patients.

The prevalence of CAM use in the Korean IBD patients has increased substantially from $60.2 \%$ in 2006 to $79.6 \%$ in $2014 .{ }^{8}$ Furthermore, $21.7 \%$ of IBD patients used CAM consistently at 2006 and 2014. This increase appears to be primarily due to increases in the prevalence of CAM use and in the frequency with which users of CAM sought professional services. The patterns and types of CAM have a wide variation between cul-

Received February 10, 2020. Accepted February 10, 2020. Correspondence to Jae Myung Cha, Department of Internal Medicine, Kyung Hee University Hospital at Gangdong, Kyung Hee University School of Medicine, 892 Dongnam-ro, Gangdong-gu, Seoul 05278, Korea. Tel: +822-440-6113, Fax: +82-2-440-6295, E-mail: drcha@khu.ac.kr ture and regions, but the most common type of CAM used in Korean IBD patients were herbal remedies in this study. In light of the observed $80.7 \%$ use of herbal remedies and $67.0 \%$ use of nutrition and diet supplements in IBD patients, it is not surprising to find that nearly 2 in 3 IBD patients was taking herbs, nutrition and diet supplements, or both. It was consistent with a previous Korean study reporting that $64.8 \%$ of IBD patients have CAM products. ${ }^{9}$ In other Korean survey, ${ }^{10}$ the most common type of CAM used were also vitamin complex (33.3\%) and red ginseng (25.0\%) in IBD patients. Therefore, physicians need to understand that IBD patients may use CAM in real world, because they experienced lack of response to conventional therapies, want to perceive favorable safety profile and a sense of greater control over their disease. ${ }^{9}$ The risk factors for CAM use included high education level, ${ }^{8,9}$ high income level, ${ }^{9}$ prior side effect to conventional therapies, ${ }^{8}$ longer duration of IBD ${ }^{9}$ and prior use of corticosteroids. ${ }^{8}$

Despite the dramatic increases in CAM use, a major concern is that the extent to which patient discloses their CAM use to their physicians remains low. Only $28.7 \%$ of CAM uses were disclosed to a physician in previous study. ${ }^{9}$ It may be risky because $13.9 \%$ of IBD patients have withdrawn their conventional therapies during CAM use. ${ }^{9}$ It would be overly simplistic to blame either the patient or their physician for this inadequacy in patient-physician communication. Shared decision mak- 
ing between physician and IBD patients need to be developed to overcome the status quo, “don't ask and don't tell." Many IBD patients may not have disclose their CAM use to their physician under the perception that CAM is not a real medication. Sometimes, it is not easy even for physician to define CAM in relationship to existing medical school curricula, clinical training and practice. For example, therapies such as lifestyle diet, vitamin, biofeedback, hypnosis, guide imagery and relaxation technique may be considered as representative of the more preventive or conventional side of medicine rather than CAM. Therefore, regular medical education should include the potential benefits and risk of CAM use in IBD patients considering their high prevalence.

IBD patients are likely to use CAM to obtain a synergistic effect or amelioration of side effects from conventional therapies. However, potential adverse interactions of CAM with conventional medications, including alterations of drug bioavailability or efficacy, should be considered. In the current issue, ${ }^{8}$ the proportion of IBD patients who perceived a positive effect after CAM use significantly decreased from $45.9 \%$ in 2006 to $33.0 \%$ in 2014. In previous study, ${ }^{9} 12.0 \%$ of CAM users experienced side effect with CAM use and $18.5 \%$ of CAM users experienced more frequent adverse effects with CAM compared with conventional therapy. Therefore, CAM should not be perceived as more effective treatment than conventional therapies. Physicians should provide negative information of CAM to their IBD patients who are trying CAM.

In this issue, significantly more IBD patients feel CAM is more expensive than conventional treatment compared to about a decade ago. ${ }^{8}$ This finding was consistent with a previous study as CAM users eventually experienced higher medical costs than conventional treatments. ${ }^{9}$ Because we have no data on the cost spent on books, classes, relevant equipment, herbs or others, ${ }^{8,9}$ the estimated cost of CAM use is likely to underrepresent the current utilization patterns. The demand for health care is sensitive to how much IBD patients must pay out-ofpocket, and most CAMs are only infrequently included in insurance benefits, and even when CAMs are covered, they tend to have high deductibles and co-payments and tend to be subject to stringent limits on the number of visits or total coverage. CAM may be introduced by third-party payers as an attractive insurance product, which may lead to a decrease in overall medical insurance cost, especially for those with less expendable income. Therefore, it is noteworthy to evaluate the magnitude of the cost for CAM use in IBD patients.

Even though the attitude toward CAM has become less fa- vorable over time, the majority of IBD patients have been experiencing CAM with an overall increase of current CAM users over time. Physicians should be aware of the high prevalence of CAM use among their IBD patients and provide shard decision making for CAM use to help their IBD patients make a more informed choice about CAM use.

\section{FINANCIAL SUPPORT}

The author received no financial support for the research, authorship, and/or publication of this article.

\section{CONFLICT OF INTEREST}

No potential conflict of interest relevant to this article was reported.

\section{AUTHOR CONTRIBUTION}

Writing and approval of final manuscript: Cha JM.

\section{ORCID}

Cha JM https://orcid.org/0000-0001-9403-230X

\section{REFERENCES}

1. Eisenberg DM, Kessler RC, Foster C, Norlock FE, Calkins DR, Delbanco TL. Unconventional medicine in the United States: prevalence, costs, and patterns of use. N Engl J Med 1993;328: 246-252.

2. Cassileth BR. Complementary therapies: the American experience. Support Care Cancer 2000;8:16-23.

3. Eisenberg DM, Davis RB, Ettner SL, et al. Trends in alternative medicine use in the United States, 1990-1997: results of a follow-up national survey. JAMA 1998;280:1569-1575.

4. Zezos P, Nguyen GC. Use of complementary and alternative medicine in inflammatory bowel disease around the world. Gastroenterol Clin North Am 2017;46:679-688.

5. Abitbol V, Lahmek P, Buisson A, et al. Impact of complementary and alternative medicine on the quality of life in inflammatory bowel disease: results from a French national survey. Eur J Gastroenterol Hepatol 2014;26:288-294.

6. Hilsden RJ, Verhoef MJ, Rasmussen H, Porcino A, DeBruyn JC. Use of complementary and alternative medicine by patients with inflammatory bowel disease. Inflamm Bowel Dis 
2011;17:655-662.

7. Rawsthorne P, Clara I, Graff LA, et al. The Manitoba Inflammatory Bowel Disease Cohort Study: a prospective longitudinal evaluation of the use of complementary and alternative medicine services and products. Gut 2012;61:521-527.

8. Lee SH, Chang K, Seo KS, et al. Changes in prevalence and perception of complementary and alternative medicine use in Korean inflammatory bowel disease patients: results of an 8-year follow-up survey. Intest Res 2020;18:192-199.

9. Park DI, Cha JM, Kim HS, et al. Predictive factors of complementary and alternative medicine use for patients with inflammatory bowel disease in Korea. Complement Ther Med 2014;22:87-93.

10. Kim SB, Park SJ, Chung SH, et al. Vaccination and complementary and alternative medicine in patients with inflammatory bowel disease. Intest Res 2014;12:124-130. 